\title{
El concepto de Nuestra América en José Martí
}

\section{The Concept Nuestra América [Our America] in José Martí}

\section{O conceito da nossa América em José Marti}

\author{
Francisca López-Civeira \\ Historiadora \\ Facultad de Filosofía e Historia \\ Universidad de La Habana \\ Cuba \\ Recibido: 4/08/2018 \\ Aceptado: 10/11/2018 \\ DOI: http://doi.org/10.15359/tdna.35-65.3
}

\section{Resumen}

El artículo analiza el proceso de construcción del concepto "Nuestra América” por José Martí así como su importancia como pilar fundamental en la estrategia continental martiana. El análisis de la realidad y de los problemas de la América Latina independiente vistos en México, Guatemala y Venezuela y el examen de la sociedad estadounidense, abrieron las posibilidades para la elaboración del concepto, el cual contenía una estrategia continental para superar los problemas internos y los peligros externos.

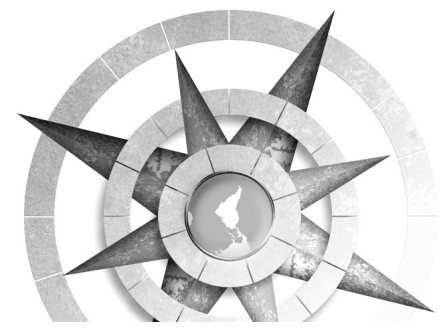

Palabras clave: Nuestra América, José Martí, estrategia latinoamericana, pensamiento latinoamericano
Abstract
The article analyzes the process of construction of the concept "Nuestra América" [Our America] by José Martí, as well as its importance as a fundamental milestone in Marti's continental strategy. The analysis of the reality and the problems of the independent Latin America seen in Mexico, Guatemala and Venezuela, as well as the examination of the North American (USA) society opened the possibilities for the elaboration of this concept, which conveyed a continental strategy for the overcoming of the internal problems and the external dangers.

Keywords: Our America, José Martí, Latin American Strategy, Latin American Thought 


\section{Resumo}

$\mathrm{O}$ artigo analisa o processo de construção do conceito "Nossa América" de José Martí, bem como sua importância como pilar fundamental na estratégia continental de Martí. A análise da realidade e dos problemas da América Latina independente vistos no México, Guatemala e Venezuela, e o exame da sociedade americana, abriram as possibilidades para a elaboração do conceito, que continha uma estratégia continental para superar problemas internos e perigos externos.

Palabras chave: Nossa América, José Martí, estratégia latino-americana, pensamento latino-americano

José Martí es uno de los mayores pensadores de la América Latina del siglo XIX. A pesar del tiempo transcurrido, sus ideas mantienen una asombrosa actualidad en el contexto del siglo XXI. Entre los asuntos más significativos del pensamiento martiano, se encuentra el concepto de Nuestra América con el cual agrupó a la región americana al sur del río Bravo. Esta construcción no fue súbita ni resultado de una inspiración puramente emotiva, sino que la fue construyendo a lo largo de la propia experiencia de Martí en diferentes escenarios y de su capacidad de ahondar en la historia y prever el posible decurso futuro.
El primer contacto de Martí con la realidad de Latinoamérica independiente fue en 1875, deportado cubano, al arribar a México; ese sería un momento capital. Su experiencia anterior se había desarrollado en su Cuba natal, todavía colonia de España y con la infame institución de la esclavitud en plena vigencia. De modo que en México se puso en contacto con un mundo nuevo, que había alcanzado la independencia y la vida republicana, metas por las que luchaba el pueblo cubano y que el adolescente Martí había abrazado, lo que le costó prisión, trabajos forzados y destierro. La entrada en México, por tanto, significaba el descubrimiento de una realidad muy nueva para él.

\section{La revelación de Nuestra América}

La tierra mexicana ofreció la posibilidad de conocer el mundo de la América, antigua colonia española, viviendo entonces un proceso de reformas liberales, dentro de un sistema republicano donde había conflictos sociales como el drama del indio americano, pugnas entre grupos políticos, debates sobre problema económicos que incluían la relación comercial con los Estados Unidos, entre los más notorios.

Uno de los primeros asuntos que observó y planteó el cubano, fue la necesidad de buscar formas propias de solución a nuestros problemas, pues apreció que se asumían fórmulas de sociedades

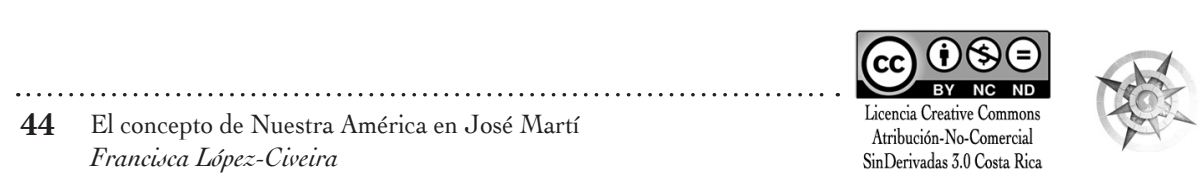


ajenas que, por sus diferencias con la realidad latinoamericana, no ofrecían solución adecuada a nuestras necesidades. Europa y Estados Unidos eran sociedades muy diferentes, por tanto, la copia servil de sus modelos no resultaba de provecho para nuestros países, de ahí que afirmara en 1875, al referirse al teatro, pero con un sentido mucho más abarcador: "Un pueblo nuevo necesita una nueva literatura. Esta vida exuberante debe manifestarse de una manera propia. [...]." A continuación, agregaba: "La vida americana no se desarrolla, brota" Y caracterizaba a nuestros pueblos "nuevos" resultantes de la mezcla de lo autóctono con lo europeo, como que "piensan de una manera que tiene más luz, sienten de una manera que tiene más amor," por lo que en el teatro debía expresarse como "brotación original de tipos nuevos" y no con copias "serviles de naturalezas agotadas" (OC., 2002, t. 6, pp. 199-200). Expresaba así, sus primeras aproximaciones al mundo americano que sentía propio y original y contrastaba con otras realidades.

La idea de pueblo nuevo volvería a estar presente en Guatemala, cuando dijo:

Interrumpida por la conquista la obra natural y majestuosa de la civilización americana, se creó con el advenimiento de los europeos un pueblo extraño, no español, porque la savia nueva rechaza el cuerpo viejo; no indígena, porque se ha sufrido la ingerencia (sic) de una civilización devastadora, dos palabras que, siendo un antagonismo, constituyen un proceso; se creó un pueblo mestizo en la forma, que con la reconquista de su libertad, desenvuelve y restaura su alma propia (OC. t. 7, p.96).

Martí arribaba así a sus primeras definiciones esenciales que, por demás, lo contraponía al entonces extendido concepto de "civilización contra barbarie" donde lo original americano era lo bárbaro. Por esto, diría en un avance de su propia definición de lo americano nuestro: "No es que las inteligencias americanas rechacen la profundidad; es que necesitan ir por un camino brillante hacia ella. / [...] la exuberancia de estos pueblos vírgenes, se manifiesta poderosamente en todas las formas". (Revista Universal, 18 de junio de 1875, en OC. t. 6, pp. 235 y 236)

El sentido de buscar formas propias, frente a la copia servil de las europeas o estadounidenses también se arraigó en el joven cubano en esos momentos iniciales, lo que se aprecia en sus frecuentes enunciados en México, como cuando dice: "A propia historia, soluciones propias. A vida nuestra, leyes nuestras"; esto lo condujo a llamar a la creación de una economía en correspondencia con la vida que se iba conformando y a promulgar leyes "originales y concretas" en armonía con los conflictos que producía 
la propia vida.(p. 312). De esta idea emanaba una muy concreta: "La imitación servil extravía, en Economía, como en literatura y en política” (p. 335).

El 26 de octubre de 1875, Martí al referirse a la necesidad de potenciar nuestras fuerzas, estableció la comparación no solo con Europa sino, también, dentro de lo que después llamaría dos factores continentales, plasmando diferencias que nos caracterizaban:

Y ¿qué fuerzas no se descubrirían en nosotros, arrojando los montones de luz de Víctor Hugo sobre nuestros ocho millones de habitantes? Y como en nosotros en toda la América del Sur. No somos aún bastante americanos: todo continente debe tener su expresión propia: tenemos una vida legada, y una literatura balbuciente. Hay en América hombres perfectos en la literatura europea; pero no tenemos un literato exclusivamente americano. Ha de haber un poeta que se cierna sobre las cumbres de los Alpes de nuestra sierra, de nuestros altivos Rocallosos; un historiador potente más digno de Bolívar que de Washington, porque la América es el exabrupto, la brotación, las revelaciones, la vehemencia, y Washington es el héroe de la calma; formidable, pero sosegado; sublime, pero tranquilo.(OC. t. 6 , p. 352).

La contraposición Washington-Bolívar resulta bien significativa, en este caso, para definir sensibilidades distintas, pueblos con características diferentes. También de su encuentro con México resultó una primera expresión que sería paradigmática: "Si Europa fuera el cerebro, nuestra América seria el corazón. Otros pensarán más, nadie sentirá mejor" (p. 423). Martí no afirmaba sino expresaba en sentido potencial la diferencia entre Europa y nuestra América, cuando por primera vez utilizaba esta expresión que habría de profundizar y desarrollar en los años posteriores. También allí, al decidir su partida, escribió el sentido de esta tierra como madre: "Y cuando yo veo a la tierra americana, hermana y madre mía, (...)" (OC. t. 6, p. 362). Al sentirla como madre, se situaba en la condición de hijo, con los deberes que eso comporta. Estas primeras expresiones, muestras del descubrimiento de nuestra América, tendrían continuidad importante en Guatemala, donde definió espacios geográficos y símbolos pertenecientes a ese mundo que comenzaba a ver como uno y propio. En Patria y Libertad. Drama indio, que escribió por solicitud para conmemorar la independencia, plasmó definiciones relevantes que sitúa en voz del personaje que nombra Martino: 
¡Soy la oveja

que se revuelve indómita ante el lobo y exánime y atónito lo deja con el arma de Maipú y Carabobo! ¡Soy de Hidalgo la voz. Soy la mirada

ardiente de Bolivar. Soy el rayo de la eterna justicia, en que abrasada América renace,

desde las fuentes donde el Bravo nace hasta el desierto bosque paraguayo! (OC. t. 18, p. 162)

Resulta muy significativa la manera en que une el proceso histórico como uno solo a través de símbolos como batallas y personajes muy representativos, al tiempo que define el espacio físico que ocupa esa, Nuestra América.

En ese descubrimiento inicial también aparece otro factor: la relación con la otra América. En México analizó el asunto del comercio bilateral, lo que mantuvo su interés en los años siguientes. En 1883 volvería sobre este tema al hablarse de un tratado comercial, acerca del cual dijo que no había "acontecimiento de gravedad mayor para los pueblos de nuestra América Latina que el tratado comercial que se proyecta entre los Estados Unidos y México." (La América, marzo de 1883, en OC., t. 7, p. 17). Consideraba que se trataba de una vecindad peligrosa y alertaba sobre ella. Esta preocupación, que se extendía para toda la América al sur del río Bravo, quedó plasmada en unas notas, al parecer escritas en un viaje de la ciudad de México a Veracruz en 1876, donde apuntó:

Sí:-descripción de lo que veo. México crece. Ha de crecer pa. Ia defensa, cuando sus vecinos crecen pa. la codicia. Ha de ser digno del mundo, cuando a sus puertas se vea librar la batalla del mundo. ¿Qué va a ser América: Roma o América, César o Espartaco? ¿Qué importa que el César no sea uno, si la nación, como tal una, es cesárea? ¡Abajo el cesarismo americano! ¡Las tierras de habla española son las que han de salvar en Am. la libertad! [...].(OC. t. 19. pp. 21-22) 1

Esta nota es muy clara acerca de la preocupación de Martí sobre los peligros que observaba dentro del continente, y también de su mirada más allá de un país, pues se dirige a toda la porción de habla española, mas es el azteca el que está más próximo a la "nación cesárea”, de ahí que exclame: "¡Oh México querido! ¡Oh México adorado, ve los peligros que te cercan! ¡Oye el clamor de un hijo tuyo que no nació de ti! Por el Norte un vecino avieso se cuaja [...]" (OC. t. 19, p. 22)

1 En las Obras Completas citadas se adjunta la siguiente anotación: "Parece que estas notas fueron tomadas por Martí en el viaje de Veracruz a Ciudad México (1875)"; sin embargo, Herrera Franyutti las ubica en 1876, cuando Martí iba de México a Veracruz. 
La consolidación de la patria común en Martí

En abril de 1877, con 24 años de edad, Martí estaba en Guatemala, donde continuó su observación de una sociedad independiente del colonialismo español, con procesos y situaciones que tenían ciertas similitudes con lo observado en México. Allí acendró la visión sobre nuestra América, no solo en su espacio geográfico y en la cultura americana nuestra, sino que incluyó nuevos aspectos.

En su análisis de los Códigos nuevos, señaló la importancia del cambio de leyes, pues "roto un estado social" había que romper las leyes para que respondieran a ese nuevo estado. De ahí que, si bien estos códigos no eran perfectos, porque tampoco lo era la sociedad en tránsito, saludaba que: “¡Al fin se es americano en América, vive republicanamente la República [...]!” (OC. t. 7, p. 102). Es decir, que era importante crear las bases de la vida republicana independiente desde el cambio que debía operarse.

En Guatemala, se percibe cómo el cubano sigue fascinado con la naturaleza americana nuestra que llama aquí "robusta”, y reitera el sentido comparativo para exaltar nuestros valores; pero lo más importante es la manera en que analiza los problemas que nos son comunes y la necesidad de transformación de las sociedades que habían alcanzado la independencia sin completar el cambio revolucionario, lo que expresa con claridad en carta de 27 de noviembre de 1877: "La manera de celebrar la independencia no es, a mi juicio, engañarse sobre su significación, sino completarla." En la propia carta a Valero Pujol, afirmaba: "Les hablo de lo que hablo siempre: de este gigante desconocido, de estas tierras que balbucean, de nuestra América fabulosa. Yo nací en Cuba, y estaré en tierra de Cuba aun cuando pise los no domados llanos del Arauco.

El alma de Bolívar nos alienta; el pensamiento americano me transporta." Este reconocimiento se acompañaba de un sentido de vida: "Vivir humilde, trabajar mucho, engrandecer a América, estudiar sus fuerzas y revelárselas, pagar a los pueblos el bien que me hacen: éste es mi oficio". (p. 110-112)

José Martí mostró gran preocupación por los problemas que dividían a la América nuestra, lo que significaba debilidad y peligro. En el folleto Guatemala, publicado en México en 1878, preguntaba acerca de esta situación: "Pero ¿qué haremos, indiferentes, hostiles, desunidos? ¿qué haremos para dar todos más color a las dormidas alas del insecto? ¡Por primera vez me parece buena una cadena para atar, dentro de un cerco mismo, a todos los pueblos de mi América!". Sin embargo, no quedaba en la interrogante y la afirmación 
de la unidad como aspiración, sino que recurría a la historia para mostrar el peligro de la desunión: "Pizarro conquistó al Perú cuando Atahualpa guerreaba a Huáscar; Cortés venció a Cuauhtémoc porque Xicotencatl lo ayudó en la empresa; entró Alvarado en Guatemala porque los quichés rodeaban a los zutujiles. Puesto que la desunión fue nuestra muerte, ¿qué vulgar entendimiento, ni corazón mezquino, ha menester que se le diga que de la unión depende nuestra vida ?" (p. 118). El modo casi dramático de mostrar lo que había significado la división en tiempos pasados era la vía para llamar la atención de la necesidad de la unión, pero no era solo el llamamiento, sino que ponía él su obra por delante, su sentido de hacer: "Para unir vivo lo que la mala fortuna desunió. Más acá ha de saberse lo que más allá se hace y se vale, más allá de la frontera chiapaneca. Las manos están tendidas; ésta es la hora." (p. 119).

Esta afirmación de lo que se proponía hacer y hacía desde los espacios que podía disponer, fue una constante en sus expresiones, que mostraban el sentido de lo que debía hacer como deber. A su amigo mexicano Manuel Mercado le escribió el 21 de septiembre de 1977: "Dar vida a la América, hacer resucitar la antigua, fortalecer y revelar la nueva”, (OC. t. 20, p. 32) como sentido de su vida, en lo cual la forja de la unidad constituía un objetivo esencial.
El año 1880 fue muy convulso en la vida de Martí. Después de su regreso a Cuba, su incorporación a la conspiración independentista, su nuevo destierro y su salida clandestina hacia Estados Unidos, y su incorporación en Nueva York al Club Revolucionario de esa ciudad, cuando en Cuba se desarrollaba una nueva conflagración por la independencia, conocida como Guerra Chiquita por su corta duración de un año aproximadamente, se entregó completamente al trabajo en aquel Club del cual llegó a ser presidente interino. Después de finalizada la contienda, en el primer semestre de 1881 pasó a residir a Caracas, a la que llamó "la Jerusalén de América” por ser la cuna de Bolívar. Sería el tercer país de nuestra América independiente en su experiencia de vida.

En aquella tierra donde, como escribió después, sin preguntar dónde se comía o se dormía, fue ante la estatua del Libertador a quien sentía que se debía querer como a un padre, continuó su análisis de nuestros problemas y posible futuro. En un discurso en el Club de Comercio de Caracas, el 21 de marzo de 1881, afirmó: "Se sabe que al poema del 1810 falta una estrofa”, (OC. t. 7, p. 284) con lo que estaba reafirmando el sentido de proceso único independentista de nuestras tierras, pues se refería a que él quiso escribir esa estrofa con la nueva gesta cubana; pero fue allí, en la carta a Fausto Teodoro de Aldrey, de 27 de 
julio de 1881, donde utilizó una de las expresiones más conocidas de Martí: "De América soy hijo: a ella me debo. Y de la América, a cuya revelación, sacudimiento y fundación urgente me consagro, ésta es la cuna” (p. 267).

Se puede advertir que, con su estancia de un semestre en Venezuela, Martí había completado un ciclo en la formación de su pensamiento y en la creación del concepto de "nuestra América" que se acompañaba de un sentido de vida, de una estrategia que contemplaba la unidad como un factor imprescindible, en la que hay la convicción del deber filial. Nuestra América es la madre por lo que los hijos tienen deberes que cumplir y Martí afirma esa certeza desde su disposición personal.

En el segundo semestre de 1881 Martí volvió a los Estados Unidos, para residir en Nueva York, casi ininterrumpidamente, hasta enero de 1895. Ese tiempo fue muy importante para la preparación de la Revolución que habría de entrar en un nuevo período de guerra como él mismo afirmó; pero fue también de extraordinario valor para su análisis de la sociedad estadounidense y, por tanto, para el completamiento de su estrategia nuestroamericana.

Desde la tierra del Norte, Martí escribió en periódicos que llegaban a países nuestros o que se publicaban allí. México, Honduras, Colombia, Argentina, entre otros, fueron países en cuya prensa aparecían los trabajos del cubano de manera constante. En 1883, en La América, que se publicaba en Nueva York, ya afirmaba:

Todo nuestro anhelo está en poner alma a alma y mano a mano los pueblos de nuestra América Latina. Vemos colosales peligros; vemos manera fácil y brillante de evitarlos; adivinamos, en la nueva acomodación de las fuerzas nacionales del mundo, siempre en movimiento, y ahora aceleradas, el agrupamiento necesario y majestuoso de todos los miembros de la familia nacional americana. Pensar es prever. Es necesario ir acercando lo que ha de acabar por estar junto. Sí no, crecerán odios; se estará sin defensa apropiada para los colosales peligros, y se vivirá en perpetua e infame batalla entre hermanos por apetito de tierras. (OC. t. 7, p. 325).

En ese trabajo, Martí volvía sobre el problema de la unidad, en términos comparativos, para mostrar nuestras distintas condiciones más favorables: "No hay en la América del Sur y del Centro como en Europa y Asia, razones de combate inevitables de razas rivales, que excusen y expliquen las guerras, y las hagan sistemáticas, inevitables, y en determinados momentos precisas. ¿Por qué batallarían, pues, sino por vanidades pueriles o por 
hambres ignominiosas, los pueblos de América? ¡Guerras horribles, las guerras de avaros!" (OC. t. 7, p. 325).

En el orden de las comparaciones insistió posteriormente, de manera reiterada. En 1891, decía en El Partido Liberal de México, que "ni con galos ni con celtas tenemos que hacer en nuestra América, sino con criollos y con indios"( $p$. 59). Mas había otros problemas que empezaban a tomar fuerza, como decía en carta a Roque Sáenz Peña en abril de 1890, al llamarlo para trabajar juntos en pos de que "confirmemos nuestra independencia antes de que se creen, como pudieran crearse, las condiciones que nos la podrían arrebatar." En este propósito vinculaba la independencia de Cuba pues "yo quiero que ella vaya, salvándose y salvando, por donde nuestra América va” (p. 398).

\section{La maduración del concepto}

Al finalizar la década del ochenta y comienzos de la del noventa, Martí había madurado sus ideas, su proyecto revolucionario y su estrategia para realizarlo; como parte de ello se puede apreciar su mirada a nuestra América, que era componente esencial de tales proyecciones. En este aspecto, es imprescindible recordar acontecimientos que lo llevaron a apresurar la formulación del cuerpo de ideas que se expresa en el ensayo Nuestra América, publicado en enero de 1891 o, previamente, su discurso conocido como Madre América, pronunciado el 19 de diciembre de 1889. La Conferencia Internacional Americana, celebrada en Washington entre 1889 y 1890, y reconocida como la primera conferencia panamericana, constituyó un momento de alarma para Martí. Le siguió la Conferencia Monetaria de 1891, que le confirmó sus aprensiones. En ocasión de la primera reunión mencionada, Martí siguió de cerca las actividades que se desplegaron, tales como el recorrido por zonas de los Estados Unidos a los delegados latinoamericanos, así como las discusiones propias del cónclave que comenzó el 2 de octubre de 1889 y culminó el 19 de abril del año siguiente. Él no era delegado, pero tenía relaciones con algunos de los presentes en aquella reunión, especialmente con Gonzalo de Quesada, el cubano que asistió como secretario del delegado argentino. En su correspondencia se refiere con insistencia al proyecto de anexión de Cuba y a los peligros que representaba aquella convocatoria norteamericana. Las páginas de periódicos continentales publicaron crónicas de alerta y de propuestas de actitudes que debían asumirse. En especial, algunas crónicas publicadas en La Nación, de Buenos Aires, resultan de sumo interés. En la del 2 de noviembre presentó con toda crudeza el problema que percibía: 
Jamás hubo en América, de la independencia acá, asunto que requiera más sensatez, ni obligue a más vigilancia, ni pida examen más claro y minucioso, que el convite que los Estados Unidos potentes, repletos de productos invendibles, y determinados a extender sus dominios en América, hacen a las naciones americanas de menos poder, ligadas por el comercio libre y útil con los pueblos europeos, para ajustar una liga contra Europa, y cerrar tratos con el resto del mundo. De la tiranía de España supo salvarse la América española; y ahora, después de ver con ojos judiciales los antecedentes, causas y factores del convite, urge decir, porque es la verdad, que ha llegado para la América española la hora de declarar su segunda independencia. (congreso Internacional de Washington", 2 de noviembre de 1889 , en OC, t. 6, p. 46).

Después de alertar con esta reflexión acerca de la intención del convite de los Estados Unidos, que incluyó los problemas en la esfera internacional; pasó a presentar lo que se podía hacer en el tiempo histórico que se vivía:

Sólo una respuesta unánime y viril, para la que todavía hay tiempo sin riesgo, puede libertar de una vez a los pueblos españoles de América de la inquietud y perturbación, fatales en su hora de desarrollo, en que les tendría sin cesar, con la complicidad posible de las repúblicas venales o débiles, la política secular y confesa de predominio de un vecino pujante y ambicioso, que no los ha querido fomentar jamás, ni se ha dirigido a ellos sino para impedir su extensión, como en Panamá, o apoderarse de su territorio, como en México, Nicaragua, Santo Domingo, Haití y Cuba, o para cortar por la intimidación sus tratos con el resto del universo, como en Colombia, o para obligarlos, como ahora, a comprar lo que no puede vender, $\mathrm{y}$ confederarse para su dominio (p. 46-47).

Era un recuento desde la historia reciente para mostrar el peligro real que se enfrentaba. Al final de la primera parte de esta crónica presentaba la comparación que permitía entender su planteamiento:

El Sun de Nueva York, lo dijo ayer: "El que no quiera que lo aplaste el Juggernaut, súbase en su carro". Mejor será cerrarle al carro el camino.

Para eso es el genio: para vencer la fuerza con la habilidad. Al carro se subieron los tejanos, y con el incendio a la espalda, como 
zorros rabiosos, o con los muertos de la casa a la grupa, tuvieron que salir, descalzos y hambrientos, de su tierra de Texas (p. 54).

La urgencia que sintió ante los intereses que se movían tras la invitación estadounidense llevó a Martí a exponer públicamente algunas de sus aprensiones que tenían como base en la existencia de dos porciones en América que eran diferentes en origen, historia e intereses, asunto de gran valor para su formulación del concepto de nuestra América:

De una parte hay en América un pueblo que proclama su derecho de propia coronación a regir, por moralidad geográfica, en el continente, y anuncia, por boca de sus estadistas, en la prensa y en púlpito, en el banquete y en el congreso, mientras pone la mano sobre una isla y trata de comprar otra, que todo el norte de América ha de ser suyo, y se le ha de reconocer derecho imperial del istmo abajo, y de otra están los pueblos de origen y fines diversos, cada día más ocupados y menos recelosos, que no tienen más enemigo real que su propia ambición, y la del vecino que los convida a ahorrarle el trabajo de quitarles mañana por la fuerza lo que le pueden dar de grado ahora. ¿Y han de poner sus negocios los pueblos de América en manos de su único enemigo, o de ganarle tiempo, y poblarse, y unirse, y merecer definitivamente el crédito y respeto de naciones, antes de que ose demandarles la sumisión el vecino a quien, por las lecciones de adentro o las de afuera, se le puede moderar la voluntad, o educar la moral política, antes de que se determine a incurrir en el riesgo y oprobio de echarse, por la razón de estar en un mismo continente, sobre pueblos decorosos, capaces, justos, y como él, prósperos y libres? (p. 56).

De ahí su pregunta a los pueblos de nuestra América: “¿A qué ir de aliados, en lo mejor de la juventud, en la batalla que los Estados Unidos se preparan a librar con el resto del mundo? ¿Por qué han de pelear sobre las repúblicas de América sus batallas con Europa, y ensayar en pueblos libres su sistema de colonización?" (p. 57).

La extensión de las citas aquí incorporadas resulta inevitable para entender los planteamientos de Martí y su urgencia de darlos a conocer, pues nada es más elocuente que sus propias palabras. Sin duda, el Congreso Internacional de Washington, fue un suceso trascendente que lo condujo a una exposición casi inmediata del concepto y de la estrategia que debía seguirse. Un primer escalón fue su discurso del 19 de diciembre ante los delegados a la Conferencia en la Sociedad Literaria Hispano-americana, en 
Nueva York, donde hizo una historia comparada entre las que ya identificaba como dos Américas y estableció el origen de las diferencias desde una mirada histórica, lo que sintetizó en la expresión: "Del arado nació la América del Norte, y la Española, del perro de presa”. (OC. t. 6, p. 136). Al final de este discurso utiliza la frase "madre América", de ahí que se le conozca por esa expresión; pero en enero de 1891 llegó al momento culminante de este proceso, cuando publicó su ensayo Nuestra América. El concepto había madurado y resultaba inaplazable su exposición.

Desde el primer párrafo ya se plantea el llamado a despertar ante el peligro "de los gigantes que llevan siete leguas en las botas" y se la pueden poner encima, de "la pelea de los cometas en el Cielo, que van por el aire dormidos engullendo mundos"; razones por las cuales eran tiempos de poner las armas de almohadas y afirma que se trata de las armas de las ideas, pues "Trincheras de ideas valen más que trincheras de piedra". ${ }^{2}$

Ante el peligro que describe desde el inicio del texto, se produce el llamado a la unidad en términos perentorios: "ilos árboles se han de poner en fila, para que no pase el gigante de las siete leguas! Es la hora del recuento, y de la marcha unida, y hemos de andar

2 El ensayo se encuentra en el Tomo 6 de la edición citada, entre las páginas 15 a 23, por ello todas las citas se remiten a esta referencia. en cuadro apretado, como la plata en las raíces de los Andes." Es evidente la intencionalidad de comenzar la exposición con una alerta y un llamado de urgencia para los lectores. A continuación expondría un conjunto de ideas, con razonamientos y explicaciones en bloques compactos donde comienza por mostrar la necesidad de la identificación de nosotros con nuestra tierra y, por tanto, crear, desde nuestra realidad, las soluciones a nuestros problemas pues "Con un decreto de Hamilton no se le para la pechada al potro del llanero. Con una frase de Sieyés no se desestanca la sangre cuajada de la raza india." Por tanto, "El gobierno ha de nacer del país. [...]." A continuación, Martí enfrenta la formulación de Sarmiento, muy extendida en aquel tiempo: "[...] No hay batalla entre la civilización y la barbarie, sino entre la falsa erudición y la naturaleza." Afirmación que se continúa con la explicación de su contenido y culmina al expresar que "Los políticos nacionales han de reemplazar a los políticos exóticos. Injértese en nuestras repúblicas el mundo; pero el tronco ha de ser el de nuestras repúblicas." Como puede observarse, no se trata de rechazar por capricho las experiencias ajenas, sino de tomar lo útil desde la base propia.

Uno de los asuntos de mayor relevancia del ensayo Nuestra América está en la mirada a la no realización del cambio revolucionario y respecto al contenido de tal cambio. Martí afirma: "El 
problema de la independencia no era el cambio de formas, sino el cambio de espíritu." Lo que se continúa con la explicación: "Con los oprimidos había que hacer causa común, para afianzar el sistema opuesto a los intereses y hábitos de mando de los opresores." Resulta muy evidente que Martí entendía que se trataba de hacer la revolución cultural, de romper con los mecanismos y mentalidades coloniales para lo cual la participación de los oprimidos resultaba fundamental, los oprimidos que nombraría después al tratar del campesino, del negro, del indio. Mas también alertaba de los peligros internos y externos, al hablar del tigre de adentro y el de afuera, de ese tigre que "vuelve de noche al lugar de la presa”, con zarpas de terciopelo, no se le oye venir, pero cuando la presa despierta "tiene al tigre encima”, alertando de los peligros internos, de cómo "la colonia continuó viviendo en la república”; pero también alertando sobre el "tigre de afuera".

Martí expuso en este ensayo lo que consideró en aquel momento, "el peligro mayor" que venía de "la diferencia de orígenes, métodos e intereses entre los dos factores continentales, y es la hora próxima en que se le acerque, demandando relaciones íntimas, un pueblo emprendedor y pujante que la desconoce y la desdeña." Para el cubano, "El desdén del vecino formidable, que no la conoce, es el peligro mayor de nuestra América". Ante ello, "urge, porque el día de la visita está próximo, que el vecino la conozca, la conozca pronto, para que no la desdeñe. [...]" Por tanto, nuestra América tenía deberes urgentes que cumplir.

La publicación Nuestra América es un momento esencial en la maduración de un concepto y una estrategia que se fue construyendo a lo largo del tiempo, las experiencias y las reflexiones de Martí, dentro de un sentido de vida, de cumplimiento del deber de hijo de la Madre América. De ahí que al estallar la guerra de independencia cubana en 1895, su gran líder se dirigiera a hombres latinoamericanos desde la perspectiva de la importancia de esa gesta para nuestra América. Al dominicano Federico Henríquez y Carvajal escribió el 25 de marzo: "Las Antillas libres salvarán la independencia de nuestra América, y el honor ya dudoso y lastimado de la América inglesa, y acaso acelerarán y fijarán el equilibrio del mundo. Vea lo que hacemos, $\mathrm{Vd}$. con sus canas juveniles,- y yo, a rastras, con mi corazón roto". (OC. t. 4, p. 111). Mientras el 18 de mayo advertía a su amigo mexicano Manuel Mercado que ya estaba en peligro de dar su vida por su país y "por mi deber-puesto que lo entiendo y tengo ánimos con que realizarlo-de impedir a tiempo con la independencia de Cuba que se extiendan por las Antillas los Estados Unidos y caigan, con esa fuerza más, sobre nuestras tierras de América." (p. 167). Al mismo tiempo preguntaba 
si México no auxiliaría a quien lo defendía para afirmar que "Esto es muerte o vida, y no cabe errar”. (p. 169).

El sentido de Nuestra América contiene una estrategia y una vocación de cumplimiento del deber hacia la tierra que se siente como madre. Constituye uno de los ejes fundamentales del pensamiento martiano que conserva absoluta actualidad para los pueblos que vivimos al sur del río Bravo, por lo que su estudio no se remite al pasado, sino que desde ese pasado se proyecta al presente y al futuro. Es un asunto de absoluta actualidad. 